\title{
Diferenças por sexos referente a ansiedade e visitas não programadas em pacientes
}

\section{hipertensos}

\author{
Gender differences in anxiety and unscheduled visits in hypertensive patients \\ Diferencias de género en ansiedad y visitas no programadas en pacientes hipertensos
}

Recebido: 07/10/2021 | Revisado: 13/01/2022 | Aceito: 15/01/2022 | Publicado: 16/01/2022

Glessiane de Oliveira Almeida

ORCID: https://orcid.org/0000-0002-1366-459X Universidade Federal de Sergipe, Brasil

E-mail: gleissi_psi@yahoo.com.br

Ikaro Daniel de Carvalho Barreto

ORCID: https://orcid.org/0000-0001-7253-806X Universidade Federal Rural de Pernambuco, Brasil E-mail: daniel.carvalho.ib@gmail.com

Felipe J. Aidar

ORCID: https://orcid.org/0000-0001-7378-4529 Universidade Federal de Sergipe, Brasil E-mail: fjaidar@gmail.com

Glaucia Regina de Oliveira Almeida

ORCID: https://orcid.org/0000-0002-0045-5887 Universidade Federal de Alagoas, Brasil E-mail: glaucia.roalmeida@gmail.com Dihogo Gama de Matos

ORCID: https://orcid.org/0000-0002-7394-8527 Universidade Federal de Sergipe, Brasil E-mail: dihogogmc@hotmail.com

Marcos Antonio Almeida-Santos ORCID: https://orcid.org/0000-0003-0622-6257 Universidade Tiradentes, Brasil

E-mail: marcosalmeida2010@yahoo.com.br Antônio Carlos Sobral Sousa

ORCID: https://orcid.org/0000-0002-4158-9726 Universidade Federal de Sergipe, Brasil E-mail: acssousa@terra.com.br

\section{Resumo}

Introdução: A hipertensão arterial sistêmica (HAS) é uma doença crônica não transmissível, multifatorial associada a fatores, como a ansiedade. A ansiedade comporta-se diferente entre homens e mulheres. Assim, o presente estudo avaliou as diferenças por sexos na ansiedade e visitas não programadas em pacientes hipertensos. Método: Estudo observacional foi realizado com 1000 voluntários hipertensos (idade: 61,0 $\pm 12,5$ ). Por meio de um questionário, foram coletados dados sociodemográficos e clínicos sobre visistas não programadas à urgência e ansiedade. A ansiedade foi avaliada pelo Inventário de Ansiedade Traço-Estado (IDATE). Resultados: Observamos que o sexo feminino é significativamente maior quanto a presença de comorbidades $(h=0,134 ; p=0,036)$, diabetes mellitus $(h=0,137 ; p=0,034)$, dislipidemia $(h=0,137 ; p=0,032)$, depressão $(h=0,240, p=0,001)$, comprar medicamentos em farmácia popular $(h=0,240$; $\mathrm{p}<0,001)$, automedicação $(h=0,200 ; \mathrm{p}=0,002)$, visita não programada e ansiedades traço $(\mathrm{h}=0,290 ; \mathrm{p}<0,001)$ e estado $(\mathrm{h}=0,239 ; \mathrm{p}<0,001)$. Na análise multivariada, o sexo masculino apresenta uma maior chance de visitas não programadas (RC: 1,43 (IC95\%: 1,07-1,92), p=0,015), e o sexo feminino maiores chances de ansiedade traço (RC: 1,59 (IC95\%: 1,21-2,09) p=0,001) e estado (RC: 1,40 (IC95\%: 1,06-1,85) p=0,016). Conclusão: Em conclusão, embora as mulheres sejam mais ansiosas, tanto Estado quanto Traço, os homens são os que mais buscam visitas não programadas à urgência. Palavras-chave: Hipertensão arterial sistêmica; Ansiedade; Urgência; Risco cardiovascular.

\begin{abstract}
Introduction: Systemic arterial hypertension (SAH) is a chronic, non-communicable, multifactorial disease associated with factors such as anxiety. Anxiety behaves differently between men and women. Thus, the present study evaluated gender differences in anxiety and unscheduled visits in hypertensive patients. Method: Observational study was carried out with 1000 hypertensive volunteers (age: $61.0 \pm 12.5$ ). Through a questionnaire, sociodemographic and clinical data on unscheduled visits to urgency and anxiety were collected. Anxiety was assessed using the State-Trait Anxiety Inventory (STAI). Results: We observed that females are significantly higher in the presence of comorbidities $(\mathrm{h}=0.134$; $\mathrm{p}=0.036)$, diabetes mellitus $(\mathrm{h}=0.137 ; \mathrm{p}=0.034)$, dyslipidemia $(\mathrm{h}=0.137 ; \mathrm{p}=0.032)$, depression $(\mathrm{h}=0.240, \mathrm{p}=0.001), \mathrm{buy}$ medication at a popular pharmacy $(h=0.240 ; \mathrm{p}<0.001)$, self-medication $(\mathrm{h}=0.200 ; \mathrm{p}=0.002)$, unscheduled visit and trait
\end{abstract}


anxieties $(h=0.290 ; p<0.001)$ and status $(h=0.239 ; p<0.001)$. In the multivariate analysis, males are more likely to have unscheduled visits (OR: 1.43 (95\%CI: 1.07-1.92), $\mathrm{p}=0.015)$, and females are more likely to have trait anxiety (OR: 1.59 (95\% CI: $1.21-2.09) \mathrm{p}=0.001)$ and state anxiety (OR: $1.40(95 \% \mathrm{CI}: 1.06-1.85) \mathrm{p}=0.016)$. Conclusion: In conclusion, although women are more anxious, both State and Trait, men are the ones who most seek unscheduled visits to the emergency room.

Keywords: Systemic arterial hypertension; Anxiety; Urgency; Cardiovascular risk.

\section{Resumen}

Introducción: la hipertensión arterial sistémica (HSA) es una enfermedad crónica, no transmisible y multifactorial asociada a factores como la ansiedad. La ansiedad se comporta de manera diferente entre hombres y mujeres. Por lo tanto, el presente estudio evaluó las diferencias de género en la ansiedad y las visitas no programadas en pacientes hipertensos. Método: Se realizó un estudio observacional con 1000 voluntarios hipertensos (edad: $61,0 \pm 12,5)$. A través de un cuestionario se recogieron datos sociodemográficos y clínicos sobre visitas no programadas a urgencias y ansiedad. La ansiedad se evaluó mediante el Inventario de ansiedad rasgo del estado (STAI). Resultados: Observamos que el sexo femenino es significativamente mayor en presencia de comorbilidades $(\mathrm{h}=0.134 ; \mathrm{p}=0.036)$, diabetes mellitus $(\mathrm{h}=0.137 ; \mathrm{p}=0.034)$, dislipidemia $(\mathrm{h}=0.137 ; \mathrm{p}=0.032)$, depresión $(\mathrm{h}=0.240, \mathrm{p}=0.001)$, comprar medicamentos en una farmacia popular $(\mathrm{h}=0.240 ; \mathrm{p}<0.001)$, automedicación $(\mathrm{h}=0.200 ; \mathrm{p}=0.002)$, visitas no programadas y ansiedad característica $(h=0.290 ; p<0.001)$ y estado $(h=0,239 ; p<0,001)$. En el análisis multivariado, los hombres tienen más probabilidades de tener visitas no programadas (OR: 1,43 (IC del 95\%: 1,07-1,92), p = 0,015) y las mujeres tienen mayores posibilidades de tener ansiedad por rasgos (OR: 1,59 (IC del 95\%: 1,21-2,09) p = 0,001) y estado (OR: 1,40 (IC 95\%: 1,06-1,85) p = 0,016). Conclusión: En conclusión, aunque las mujeres son más ansiosas, tanto de Estado como de Rasgo, los hombres son los que más buscan visitas no programadas a urgencias.

Palabras clave: Hipertensión arterial sistémica; Ansiedad; Urgencia; Riesgo cardiovascular.

\section{Introdução}

A hipertensão arterial sistêmica representa o fator de risco isolado mais importante de causa de morte por doenças cardiovasculares (Malachias, et al., 2016) devido à dificuldade de controle (Magnabosco, et al., 2017). Estima-se que em 2025, no Brasil, a prevalência de HAS será de 40\% (Silva, et al., 2016). Os principais fatores de risco para a HAS são a idade, sexo, sobrepeso, raça, predisposição genética, obesidade e hábitos pouco saudáveis como não realizar atividade física, consumo excessivo de sal, bebidas alcoólicas, tabagismo, estresse e a ansiedade (Malachias, et al., 2016).

Hipertensão e transtornos de ansiedade causam morbidade e custo alto para o sistema de saúde, além de ser um assunto de controvérsia com resultados distintos em estudos observacionais e populacionais de grande escala (Januzzi, et al., 2000; Wu, et al., 2014; Aragão, et al., 2017). No Brasil, o transtorno de ansiedade equivale 9,3\% da população geral, representando o pior resultado se comparado a todos os países do mundo (World Health Organization, 2017).

A literatura apresenta dados sobre a prevalência da HAS e fatores de risco, principalmente associados a transtornos mentais, entre homens e mulheres (Silva, et al., 2016; Silva, et al., 2016), com um risco 3 vezes maior de doenças cardiovasculares se comparados com a população em geral, em um período de 10 anos (Wu, et al., 2014). Em uma publicação de Feng et al. (2016), demonstrou que as mulheres apresentavam o maior risco de ansiedade em relação à doença cardiovascular. Atualmente, a inserção da mulher, como força de trabalho, em diferentes contextos na sociedade, além de continuar desenvolvendo seu papel dentro do contexto familiar favorece o aparecimento de sinais e sintomas físicos e psíquicos (Silva, et al., 2016).

Em um estudo realizado por Carvalho et al. (2016), demonstrou que foi encontrado sintomas de depressão em 17,5\% dos pacientes adultos com doença cardiovascular e sintomas de ansiedade em 32,5\%, e entre estes, porém, a maior prevalência de distúrbios mentais foi associada ao sexo feminino (ansiedade: $\mathrm{p}=0,002$; depressão: $\mathrm{p}=0,022$ ). Aspectos psicossociais estão diretamente relacionadas com a hipertensão em homens e mulheres sendo tanto fator de risco independente para o não controle da pressão arterial, como consequência direta do evento cardiovascular (Tofler, et al., 2020; Almeida, et al., 2021). Dessa forma, o presente estudo teve como objetivo avaliar as diferenças por sexo referente a ansiedade e visitas não programadas em pacientes hipertensos. 


\section{Metodologia}

\subsection{Desenho do estudo}

O presente estudo caracterizou-se como transversal e observacional, pois essa abordagem permite ao pesquisador utilizar o contexto sociocultural do ambiente observado para explicar o comportamento, além de envolver, a definição de uma população de interesse com presença ou ausência de desfecho (Marietto, 2018, Gordis, 2004), realizado entre junho de 2017 e outubro de 2019 na cidade de Aracaju-Sergipe, Brasil. Este é parte da pesquisa ANOPAR - Autoaferição não orientada da pressão arterial: estudo metodológico (Almeida, et al., 2020), estudo aprovado pelo Comitê de Ética em Pesquisa envolvendo seres humanos sob o número CAAE: 60473316.9.0000.5546.

\subsection{Pacientes}

A amostra foi realizada de forma não aleatória com 1000 (mil) pacientes selecionados consecutivamente para minimizar o viés de amostragem, na cidade de Aracaju-Sergipe, Brasil. Como critério de inclusão, foram definidos pacientes maiores de 18 anos, de ambos os sexos e com diagnóstico de hipertensão arterial sistêmica, acompanhados ambulatoriamente em três instituições hospitalares da cidade de Aracaju-Sergipe, Brasil. Uma dessas instituições atende exclusivamente usuários do serviço público de saúde e duas atendem à iniciativa privada. As instituições escolhidas são referência no tratamento de pacientes hipertensos na cidade. Foram excluídos aqueles com transtornos mentais que comprometessem as respostas aos questionários ou os que recusaram a participar. Os pacientes que consentiram em participar do presente estudo assinaram o Termo de Consentimento Livre e Esclarecido.

\subsection{Procedimento}

A coleta de dados foi realizada por meio da aplicação de um questionário específico abrangendo os seguintes tópicos: (1) Identificação do paciente e elementos sociodemográficos (sexo, idade, renda, escolaridade, estado civil, automedicação, comorbidades, compra de medicamento, visitas não programadas ao pronto-socorro). A classe social e a educação foram inseridas no questionário de acordo com a classificação utilizada pelo Instituto Brasileiro de Geografia e Estatística (IBGE). O IBGE é um instituto público da administração federal brasileira, que fornece as informações geográficas e estatísticas do Brasil. O que classifica a classe social pelo grupo de renda familiar. Em que a classe A corresponde a acima de 20 salários mínimos; B: 10 a 20 salários mínimos; C: 4 a 10 salários mínimos; D: 2 a 4 salários mínimos; E: Até 2 salários mínimos. Escolaridade classificada como: ensino fundamental, médio, universitário, programa de graduação e nunca estudado; (2) Comorbidades: A identificação das comorbidades dos pacientes foram realizadas a partir da: (I) comorbidade referida: o próprio paciente referiu ter as comorbidades já diagnósticadas e em tratamento; (II) A partir do prontuário médico: O médico responsável, a partir do prontuário, confirmou o diagnóstico de comorbidades de cada paciente. (3)Ansiedade: o IDATE foi aplicado por um psicólogo treinado a todos os voluntários da pesquisa. O IDATE tem como objetivo avaliar a ansiedade como característica do estado (E) e da personalidade (T). Trata-se de um instrumento de autoavaliação, composto por duas escalas paralelas, cada uma com 20 itens (Biaggio \& Natalício, 1979). No IDATE, na escala T de acordo com a peneira, a média estipulada é de 45,34 a 55,22 e na escala $\mathrm{E}$ a média esperada é de 43,64. A consistência interna de ambas as escalas foi determinada com base no alfa de Cronbach. A escala E mostrou um alfa de Cronbach de 0,91, enquanto a escala $\mathrm{T}$ foi de 0,894. Assim, os instrumentos apresentaram alta validade interna quando comparados à população geral, com índice entre 5,6\% e 1,8\%. Valores acima de 0,8 indicam alta consistência, embora coeficientes acima de 0,60 tenham demonstrado consistência adequada (Biaggio \& Natalício, 1979). Os escores de IDATE maiores que 42 indicam ansiedade (Spielberger, et al., 1970). 


\subsection{Análise estatística}

As variáveis contínuas foram descritas como média e desvio padrão. Quanto às variáveis categóricas, foram utilizadas frequências absolutas e percentuais e intervalos de confiança de 95\% para sumarizá-las, quando relevantes. O teste de ShapiroWilk foi usado para avaliar a suposição de normalidade. Para testar as hipóteses relacionadas às variáveis categóricas, o teste do $\chi^{2}$ de Pearson foi usado. A comparação entre os sexos foi realizada por meio do teste $t$ de Student para dados independentes no caso de variáveis quantitativas. Para analisar se o sexo era um fator associado aos desfechos (visitas não programadas e ansiedade traço e estado), a técnica de regressão logística foi usado onde calculamos razões de chances brutas e ajustados para idade, hospital, situação marital, classe social, escolaridade, comorbidades, forma de compra de medicamentos e auto-medicação. Utilizamos o software R Core Team 2021 (Versão 4.1.0) em todas as análises. As estimativas foram feitas com os seguintes parâmetros: poder $=80 \%$. Os tamanhos do efeito ( $\mathrm{d}$ de Cohen para variáveis contínuas e $\mathrm{h}$ de Cohen para variáveis categóricas) foram definidos como pequenos $(<0,20)$, médios (entre 0,20 e 0,50), grandes (entre 0,50 e 0,80$)$ e muito grandes $(>1,20)($ Cohen, 1988). O valor de $\mathrm{p}$ bicaudal inferior a 0,05 foi considerado o critério de significância estatística.

\section{Resultados}

Os pacientes tinham média de idade de 61,0 \pm 12,5 anos, com mínimo de 27 anos e máximo de 100 anos. Observou-se que 50\% dos pacientes foram acompanhados no ambulatório de cardiologia do Hospital Universitário da Universidade Federal de Sergipe e a outra metade na rede suplementar. As características básicas para homens e mulheres são apresentadas na Tabela 1. O sexo feminino é estatísticamente mais associado a ser viúva, classe E e níveis mais altos de escolaridade. O sexo masculino, por outro lado, é estatísticamente mais associado a ser casado, classe social A e ter apenas ensino médio.

Tabela 1. Características gerais para homens e mulheres.

\begin{tabular}{|c|c|c|c|c|}
\hline Características Sociodemográficas & $\begin{array}{c}\text { Feminino } \\
(n=571)\end{array}$ & $\begin{array}{c}\text { Masculino } \\
(n=429)\end{array}$ & $\mathbf{P}$ & $\begin{array}{c}\text { Tamanho do } \\
\text { efeito }\end{array}$ \\
\hline Idade (anos), média \pm DP & $61,47 \pm 12,49$ & $60,42 \pm 12,41$ & 0,184 & $0,085^{\mathrm{d}}$ \\
\hline$<45, \mathrm{n}(\%)$ & $62(10,9)$ & $47(11)$ & & $-0,003^{h}$ \\
\hline $45-54$ & $99(17,3)$ & $80(18,6)$ & & $-0,034^{\mathrm{h}}$ \\
\hline $55-64$ & $162(28,4)$ & $129(30,1)$ & 0,787 & $-0,037^{\mathrm{h}}$ \\
\hline$>64$ & $248(43,4)$ & $173(40,3)$ & & $0,063^{\mathrm{h}}$ \\
\hline \multicolumn{5}{|l|}{ Hospital, n (\%) } \\
\hline Público & $299(52,4)$ & $201(46,9)$ & \multirow{2}{*}{0,085} & $0,110^{\mathrm{h}}$ \\
\hline Privado & $272(47,6)$ & $228(53,1)$ & & $-0,110^{\mathrm{h}}$ \\
\hline \multicolumn{5}{|l|}{ Estado civil, n (\%) } \\
\hline Casado & $315(55,2)$ & $292(68,1)$ & \multirow[t]{5}{*}{$<0,001$} & $-0,266^{\mathrm{h}}$ \\
\hline Divorciado & $84(14,7)$ & $49(11,4)$ & & $0,098^{\mathrm{h}}$ \\
\hline Solteiro & $85(14,9)$ & $54(12,6)$ & & $0,067^{\mathrm{h}}$ \\
\hline Viúvo & $71(12,4)$ & $20(4,7)$ & & $0,285^{h}$ \\
\hline Mora com companheiro & $16(2,8)$ & $14(3,3)$ & & $-0,027^{\mathrm{h}}$ \\
\hline \multicolumn{5}{|l|}{ Classe social, $\mathrm{n}(\%)$} \\
\hline A & $34(6)$ & $44(10,3)$ & \multirow{5}{*}{0,002} & $-0,159^{h}$ \\
\hline B & $65(11,4)$ & $66(15,4)$ & & $-0,118^{h}$ \\
\hline $\mathrm{C}$ & $95(16,6)$ & $85(19,8)$ & & $-0,082^{\mathrm{h}}$ \\
\hline $\mathrm{D}$ & $143(25)$ & $100(23,3)$ & & $0,040^{\mathrm{h}}$ \\
\hline $\mathrm{E}$ & $234(41)$ & $134(31,2)$ & & $0,203^{h}$ \\
\hline \multicolumn{5}{|l|}{ Escolaridade, n (\%) } \\
\hline Nunca estudou & $178(31,2)$ & $113(26,3)$ & \multirow{5}{*}{0,006} & $0,107^{\mathrm{h}}$ \\
\hline Fundamental & $173(30,3)$ & $142(33,1)$ & & $-0,060^{\mathrm{h}}$ \\
\hline Ensino Médio & $136(23,8)$ & $135(31,5)$ & & $-0,171^{\mathrm{h}}$ \\
\hline Superior & $29(5,1)$ & $12(2,8)$ & & $0,119^{h}$ \\
\hline Pós-Graduação & $55(9,6)$ & $27(6,3)$ & & $0,124^{\mathrm{h}}$ \\
\hline
\end{tabular}

Idade expressa em média e desvio padrão (DP); outros dados expressos em números absolutos e porcentagem entre parênteses; p: significância estatística (teste do $\chi^{2}$ de Pearson e teste $t$ de Student). A = classe social alta, B = classe média alta. $\mathrm{C}=\mathrm{Classe}$ social média. $\mathrm{D}=$ classe média baixa. $\mathrm{D}=$ Classe social pobre. Tamanho do efeito $d$ de Cohen para variáveis contínuas e $h$ de Cohen para variáveis categóricas. * p $<0,05$. Fonte: Autores. 


\subsection{Comparação entre pacientes hipertensos feminino e masculino}

Os dados dos pacientes dos sexos feminino e masculino em relação às características clínicas são apresentados na Tabela 2. Sexo feminino é significativamente maior quanto a presença de comorbidades, diabetes mellitus, dislipidemia, depressão, uso de medicação para comorbidades, comprar medicamentos em farmácia popular, automedicação e ansiedades traço e estado. O sexo masculino é significativamente maior quanto a presença de doença arterial coronariana, visita não programada e comprar medicamentos em farmácia (valor integral).

Tabela 2. Comparação entre pacientes femininos e masculinos quanto às características clínicas.

\begin{tabular}{|c|c|c|c|c|}
\hline Características Clínicas, n (\%) & $\begin{array}{c}\text { Feminino } \\
(\mathrm{n}=571)\end{array}$ & $\begin{array}{c}\text { Masculino } \\
(\mathrm{n}=\mathbf{4 2 9})\end{array}$ & $\mathbf{P}$ & $\begin{array}{c}\text { Tamanho do } \\
\text { efeito }\end{array}$ \\
\hline Comorbidades & $315(55,2)$ & $208(48,5)$ & $0,036^{*}$ & 0,134 \\
\hline Diabetes mellitus & $137(24)$ & $79(18,4)$ & $0,034 *$ & 0,137 \\
\hline Dislipidemia & $210(36,8)$ & $130(30,3)$ & $0,032 *$ & 0,137 \\
\hline Doença arterial coronária & $56(9,8)$ & $60(14)$ & $0,041 *$ & $-0,130$ \\
\hline Doença renal crônica & $11(1,9)$ & $9(2,1)$ & 0,848 & $-0,012$ \\
\hline Acidente vascular & $27(4,7)$ & $19(4,4)$ & 0,823 & 0,014 \\
\hline Depressão & $20(3,5)$ & $2(0,5)$ & $0,001 *$ & 0,240 \\
\hline Insuficiência cardíaca & $22(3,9)$ & $23(5,4)$ & 0,255 & $-0,072$ \\
\hline Doença de obstrutiva pulmonar crônica & $12(2,1)$ & $4(0,9)$ & 0,145 & 0,098 \\
\hline Doença arterial obstrutiva periférica & $15(2,6)$ & $10(2,3)$ & 0,767 & 0,019 \\
\hline Uso de medicamento para comorbidades & $280(49)$ & $184(43)$ & 0,058 & 0,121 \\
\hline \multicolumn{5}{|l|}{ Compra do medicamento } \\
\hline Posto de Saúde (gratuito) & $197(34,5)$ & $126(29,4)$ & $<0,001 *$ & 0,110 \\
\hline Farmácia Popular & $152(26,6)$ & $72(16,8)$ & & 0,240 \\
\hline Farmácia (Valor integral) & $222(38,9)$ & $231(53,8)$ & & $-0,301$ \\
\hline Automedicação & $251(44)$ & $162(37,8)$ & $0,049 *$ & 0,126 \\
\hline Visitas à urgência & $243(42,6)$ & $288(67,1)$ & $0,002 *$ & 0,200 \\
\hline \multicolumn{5}{|l|}{ Ansiedade } \\
\hline Traço & $330(57,8)$ & $186(43,4)$ & $<0,001 *$ & 0,290 \\
\hline Estado & $290(50,8)$ & $167(38,9)$ & $<0,001 *$ & 0,239 \\
\hline
\end{tabular}

Dados expressos em números absolutos e porcentagem entre parênteses; p: significância estatística (teste $\chi^{2}$ de Pearson). Tamanho do efeito $h$ de Cohen. $* \mathrm{p}<0,05$. Fonte: Autores.

Foram estimadas as razões de chance brutas e ajustadas para visita não programada, ansiedade traço e estado superior a ponto de corte em relação ao sexo, ajustando para idade, hospital, estado civil, classe social, escolaridade, comorbidades, como comprou medicamento para comorbidade e auto-medicação (Tabela 3). Observou-se que o sexo masculino é um fator associado a visita não programada, enquanto que o sexo feminino é associado a ansiedade traço e estado nos modelos brutos e ajustados.

Tabela 3. Razão de chance para ansiedade e visita não programada entre pacientes do sexo feminino em comparação com pacientes do sexo masculino.

\begin{tabular}{|c|c|c|c|c|c|c|}
\hline \multirow[b]{2}{*}{ Modelo } & \multicolumn{2}{|c|}{ Ansiedade Traço } & \multicolumn{2}{|c|}{ Ansiedade Estado } & \multicolumn{2}{|c|}{ Visitas à urgência } \\
\hline & $\begin{array}{c}\text { Feminino } \\
\text { RC } \\
(95 \% \mathrm{CI})\end{array}$ & $\mathbf{P}$ & $\begin{array}{c}\text { Feminino } \\
\text { RC } \\
(95 \% \text { CI })\end{array}$ & $\mathbf{P}$ & $\begin{array}{c}\text { Masculino } \\
\text { RC } \\
(95 \% \text { CI })\end{array}$ & $\mathbf{P}$ \\
\hline Não ajustado & $\begin{array}{c}1,79 \\
(1,38-2,30)\end{array}$ & $<0,001$ & $\begin{array}{c}1,61 \\
(1,24-2,08)\end{array}$ & $<0,001$ & $\begin{array}{c}1,51 \\
(1,16-1,96)\end{array}$ & 0,002 \\
\hline Ajustado & $\begin{array}{c}1,59 \\
(1,21-2,09)\end{array}$ & 0,001 & $\begin{array}{c}1,40 \\
(1,06-1,85)\end{array}$ & 0,016 & $\begin{array}{c}1,43 \\
(1,07-1,92)\end{array}$ & 0,015 \\
\hline
\end{tabular}

95\% IC = intervalo com 95\% de confiança. RC - Razão de Chances. Modelo ajustado para idade, hospital, estado civil, classe social, educação, comorbidades, compra do medicamento, e automedicação. * p<0,05. Fonte: Autores.

\section{Discussão}

As potenciais diferenças entre homens e mulheres hipertensos têm sido objeto de investigação por vários estudos (Serpytis, et al., 2018, Silva, et al., 2016, Silva et al., 2016). Nosso estudo demonstra que, apesar das diferenças aparentes entre 
homens e mulheres, estas são mais ansiosas que os homens. Porém, os homens são os que mais buscam visitas não programadas à urgência.

Entre os transtornos psiquiátricos, os tanstornos de ansiedade são os mais prevalentes na população em geral, com taxas de prevalência entre 5,6\% e 18,1\% (Desousa, et al., 2013). Um estudo recente de Serpytis et.al. (2018) revelou que as mulheres tinham um risco bastante elevado de apresentar distúrbio de ansiedade. Outros estudos mostraram que a prevalência de ansiedade é maior em mulheres que em homens, sendo um fator de risco para aumento da pressão arterial (Costa, et al., 2014, Silva et al., 2016, Lacerda, et al., 2017, Souza, et al., 2018).

O contexto socioeconômico das mulheres influenciam na prevalência de ansiedade. Na nossa pesquisa há predominância de mulheres que perderam seus companheiros, e representam a classe social mais baixa. Dessa forma, a dinâmica dos gêneros nas relações de poder, reflete na pouca valorização do papel da mulher na sociedade, mesmo com o aumento da participação dessas mulheres no mercado de trabalho, além das desigualdades sociais se comparado com os homens, configurando fatores influenciadores que resultam na baixa autoestima e menor controle sobre o ambiente social (Silva, et al., 2016).

Nesse contexto, eventos produtores de estresse provocam alterações no sistema endócrino, e consequentemente, aumentam o risco de desenvolvimento de distúrbios mentais (Lima \& Araújo, 2020). Partindo do pressuposto de que as mulheres, pela sua própria condição fisiológicas e psicológicas são influenciadas pela diminuição da produção de estrogênios, alterações no perfil lipídico, insônia, cansaço e irritabilidade, tornam-se mais propensas a ansiedade que os homens (Silva, et al., 2016).

A presença de comorbidades, na nossa pesquisa, foi distinta nas mulheres, quando comparadas ao sexo masculino, pela maior presença de diabetes mellitus, dislipidemia e doença pulmonar obstrutiva crônica, além disso, o maior índice de automedicação foi verificado nas mulheres. Nossos resultados corroboram os Dados da Pesquisa Nacional de Acesso, Uso e Promoção do Uso Racional de Medicamentos no Brasil (PNAUM) que apresenta que o sexo feminino tem maior influência na prática da automedicação (Mengue, et al., 2016). A Associação Brasileira das Indústrias Farmacêuticas (ABIFARMA), aponta que no Brasil cerca de 80 milhões de pessoas fazem uso de automedicação e aproximadamente 20 mil mortalidades em decorrência dessa prática. Uma das principais causas da automedicação é a necessidade de aliviar os sintomas (Naves, et al., 2010) e fatores psicossociais (Mengue, et al., 2016).

Em estudos (Gomes, et al., 2007, Moreira et al., 2011, Hachem, et al., 2018) que analisaram o perfil dos indivíduos que procuram os serviços de saúde no país, observou-se que há um predomínio de usuários que procuram atendimento de urgência com doenças crônicas, como hipertensão arterial, com maior gravidade pelo descontrole da condição. A nossa pesquisa demosntra que os homens são os que mais buscam o serviços de urgência. Homens tendem a ser menos preocupados com a saúde, têm um estilo de vida pouco saudável e são menos propensos a se perceberem como estando em risco (Wu, et al., 2014). Segundo Gomes, Nascimento \& Araújo (2007), o imaginário social de que o homem é invulnerável a doenças dificulta a sua busca por consultas médicas programadas, fazendo com que os homens não tenham, na sua rotina, o acompanhamento médico.

Sendo a hipertensão uma doença crônica e considerando-se a ansiedade um dos fatores etiológicos da doença, é possível sugerir que homens e mulheres hipertensos, busquem conhecer a doença para que as condições limitantes e frustrações promovidas pela situação da doença não agrave o seu quadro clínico, gerando um ciclo vicioso. Surge daí, a necessidade de se atentar para esse fator de risco, a ansiedade, a fim de se instituírem estratégias de manejo e enfrentamento para essa população.

\section{Conclusão}

As mulheres são mais ansiosas que os homens. Porém, os homens são os que mais buscam visitas não programadas à urgência. Assim, a falta de conhecimento sobre a doença e suas implicações, bem como as orientações erradas podem agravar o quadro clínico do paciente. Portanto, é aconselhável a busca por conhecimento sobre a doença. Além de investir em políticas públicas voltadas aos capacitores, informar a população sobre as implicações, formas de tratamento e apontar a importância da 
abordagem multidisciplinar no manejo do paciente hipertenso com transtorno de ansiedade.

Contudo, observa-se a necessidade de novos estudos longitudinais para que os pacientes sejam reavaliados em um período mais longo e contínuo, e assim determinar a associação de longo prazo entre ansiedade e visitas não programadas à urgência. Além, de avaliar a qualidade de vida de pacientes hipertensos relacionando a diferença de sexos.

\section{Agradecimentos}

Este trabalho teve o apoio da Coordenação de Aperfeiçoamento de Pessoal de Nível Superior / Fundação de Amparo à Pesquisa e Inovação Tecnológica do Estado de Sergipe.

\section{Referências}

Almeida, G. O., Aidar, F. J., Matos, D. G., Almeida-Neto, P. F., Melo, E. V., Filho, J. A. S. B., Almeida-Santos, M. A., Oliveira, V.B., Almeida, R.R., Santos, S. M., Pereira, L. M. C., Barbosa, J. S. \& Sousa, A. C. S. (2021). Non-Targeted Self-Measurement of Blood Pressure: Association with Self-Medication, Unscheduled Emergency Visits and Anxiety, Medicina (Kaunas), 57(1):75.

Almeida, G. O., Aidar, F. J., Matos, D. G., Almeida-Neto, P. F., Oliveira, V. B., Barreto, I. D. C., Almeida, R. R., Almeida-Santos, M. A., Filho, J. A. S. B. \& Sousa, A. C. S. (2020). MBPPG-Self-measurement of blood pressure without previous guidance: methodological study, Motricidade. 16, 156-160.

Aragão, E. I. S., Portugal, F. B., Campos, M. R., Lopes, C. S., Fortes S. L. C. L. (2017). Distintos padrões de apoio social percebido e sua associação com doenças físicas (hipertensão, diabetes) ou mentais no contexto da atenção primária. Ciência \& Saúde Coletiva, 22(7), $2367-2374$.

Biaggio, A. M. B. \& Natalício, L. (1979). Manual para o Inventário de Ansiedade Traço-Estado (IDATE). Centro Editor de Psicologia Aplicada-CEPA, Rio de Janeiro, Brasil.

Carvalho, I. G., Bertolli, E. D., Paiva, L., Rossi, L. A., Dantas, R. A. \& Pompeo, D. A. (2016). Anxiety, depression, resilience and self-esteem in individuals with cardiovascular diseases. Rev. Lat. Am. Enf. 24, e2836.

Cohen, J. (1988). Statistical power analysis for the behavioral sciences, Hillsdale, Lawrence Erlbaum, Assocates.

Costa, M. G. S., Dimenstein, M. D. B. \& Leite, J. F (2014). Condições de vida, gênero e saúde mental entre trabalhadoras rurais assentadas, Estud. Psicol. 19, $145-54$.

Desousa, D. A., Moreno, A. L., Gauer, G., Manfro, G. G., Koller, S. H. (2013). Revisão sistemática de instrumentos para avaliação de ansiedade na população brasileira, Avaliação Psicológica. 12, 397-410.

Feng, H. P., Chien, W. C., Cheng, W. T., Chung, C. H., Cheng, S. M., Tzeng, W. C. (2016). Risk of anxiety and depressive disorders in patients with myocardial infarction: A nationwide population-based cohort study, Medicine (Baltimore), 95 (34). e4464.

Gordis L. (2004). Epidemiology. Philadelphia, Elsevier Saunders.

Gomes, R., Nascimento, E. F., Araújo, F. C. (2007). Por que os homens buscam menos os serviços de saúde do que as mulheres? As explicações de homens com baixa escolaridade e homens com ensino superior, Cadernos de Saúde Pública, 23, 565-574.

Hachem, M. E., Siddiqui, M., Thomas, S. J., Dudenbostel, T., Valaiyapathi, B., Judd, E., Patel, P., Gupta, P., Tomaszewski, M., Oparil, S., Calhoun, D. A. (2018). Hypertensive Medications is Associated with Higher BP and Anxiety Levels, Hypertension 72, 369-374.

Januzzi Jr. J. L., Stern, T. A., Pasternak, R. C., DeSanctis, R. W. (2000). The influence of anxiety and depression on outcomes of patients with coronary artery disease, Arch. Intern. Med. 160, 1913-21.

Lacerda, M. S., Cirelli, M. A., Barros, A. L. B. L. \& Lopes, J. L. (2017). Ansiedade, estresse e depressão de familiares de pacientes com insuficiência cardíaca, Revista da Escola de Enfermagem da USP. 51, 1-8.

Lima, L. P. \& Araujo, A. L. C. (2020). A influência da desregulação hormonal no desenvolvimento de transtornos psicopatológicos. Revista Multidisciplinar Em Saúde, 1(2), 15.

Malachias, M. V. B., Barbosa, E. C. D., Martim, J. F. V., Rosito, G. B. A., Toledo, J. Y. \& Passarelli-Júnior O. (2016). 7ª DiretrizBrasileira de Hipertensão Arterial: Capítulo 14-CriseHipertensiva, Arquivo Brasileiro de Cardiologia. 107, 79-83.

Magnabosco, P., Oliveira, E. M., Toneti, A. N., Anjos, A. C. Y. \& Marchi-Alves, L. M. (2017). Prevalência e controle da hipertensão arterial: estudo comparativo entre população urbana e rural. Rev. Min. Enferm. 21, e-999.

Marietto, M. L. (2018). Observação Participante E Não Participante: Contextualização Teórica e Sugestão de Roteiro para aplicação sos Métodos. Revista IberoAmericana de Estratégia - RIAE. 17 (3), 5-18.

Mengue, S. S., Bertoldi, A. D., Boing, A. C., Tavares, N. U. L., Dal Pizzo, T. S., Oliveira, M. A., Arrais, P. S. D., Ramos, L. R., Farias, M. R., Luiza, V. L., Bernal, R. T. I. \& Barros, A. J. D. (2016). Pesquisa Nacional sobre Acesso, Utilização e Promoção do Uso Racional de Medicamentos (PNAUM): métodos do inquérito domiciliar, Rev. Saude Publica. 50, 1-13. 
Research, Society and Development, v. 11, n. 2, e0611221510, 2022

(CC BY 4.0) | ISSN 2525-3409 | DOI: http://dx.doi.org/10.33448/rsd-v11i2.21510

Moreira, J. P. L., Moraes, J. R. \& Luiz, R. R. (2011). Use of medical appointments and systemic arterial hypertension in urban and rural areas of Brazil, according to data from PNAD 2008, Rev. Ciência Saúde 16, 3781-3793.

Naves, J. O. S., Castro, L. L. C., Carvalho, C. M. S. \& Merchán-Hamann, E. (2010). Automedicação: uma abordagem qualitativa de suas motivações, Ciência Saúde Coletiva. 15 (2010) 1751-62.

Serpytis, P., Navickas, P., Lukaviciute, L., Navickas, A., Aranauskas, R., Serpytis, R., Deksnyte, A., Glaveckaite, S., Petrulioniene, Z. \& Samalavicius, R. (2018). Diferenças por Sexo na Ansiedade e Depressão após Infarto Agudo do Miocárdio, Arq. Bras. Cardiol. 111, 676-683.

Silva, E. C., Martins, M. S. A. S., Guimarães, L. V., Segri, N. J., Lopes, M. A. L. \& Espinosa, M. M. (2016). Hypertension prevalence and associated factors in men and women living in cities of the Legal Amazon, Rev. Bras. Epidemiol. 19, 38-51.

Silva, S. S. B. E., Oliveira, S. F. S. B. \& Pierin, A. M. G. (2016). O controle da hipertensão arterial em mulheres e homens: umaanálisecomparativa, Rev. Esc. Enfermagem. USP. 50, 50-58.

Souza, G. N. P., Alves, R. J. R., Souza, L. P. S. \& Rosa, A. J. (2018). Prevalência de sintomas depressivos e/ou ansiosos em pessoas com hipertensão arterial sistêmica e/ou diabetes mellitus, Revista Portuguesa de Enfermagem de Saúde Mental 20, 43-50.

Spielberger, C. D., Gorsuch, R. L., \& Lushene, R. E. (1970). Manual for the State-Trait Anxiety Inventory, Consulting Psychologist Press, Palo Alto.

Tofler, G., Silver, J. \& Salomon, D. (2020). Psychosocial factors in acute coronary syndrome. https://www.uptodate.com.

World Health Organization (2017). Depression and other common mental disorders: global health estimates. Genebra: WHO.

Wu, E. L., Chien, I. C. \& Lind, C. H. (2014). Increased risk of hypertension in patients with anxiety disorders: A population-based study, Journal of Psychosomatic Research 77, 522-527. 